\title{
Influence of Lateral-torsional Coupling Vibration on SINS Lever-arm Error Compensation
}

\author{
Qi Yang ${ }^{1,2, a)}$, Xinxue Liu ${ }^{1, \text { b) }}$, Shaofei Meng ${ }^{1, c)}$, Qingbao Liu ${ }^{2, \text { d) }}$ \\ ${ }^{1}$ Xi’an Hi-Tech Institute, Xi’an 710025, China. \\ ${ }^{2}$ Qingzhou Hi-Tech Institute, Qingzhou 262500, China. \\ a)epgc_yangqi@163.com, ${ }^{\mathrm{b}}$ LiuXX@126.com, ${ }^{\mathrm{c})}$ shaofeiM@163.com, ${ }^{\mathrm{d}}$ fuff007@163.com
}

Keywords: Coupling Vibration, SINS, Error Compensation

\begin{abstract}
The influence of complex dynamic environment in apparatus cabin for strapdown inertial navigation system(SINS) is one of the technical problems to improve navigation accuracy. The SINS was equivalent to Timoshenko-beam hybrid system, which was considered under the lateraltorsional coupling vibration. The system motion was described by dynamic differential equations, decoupled and solved by the Timoshenko-beam's Green function. The vibration response of the SINS would make the lever arm vector changed and incomplete compensation, at the same time, vibration response also introduced measurement error. Finally, with the flight simulation, the influence of lever arm error incomplete compensation and measurement error were analysed separately.
\end{abstract}

\section{Introduction}

The inertial measurement unit(IMU) usually install in the apparatus cabin of flight vehicle, which is in a rocking vibration and shock environment. The complex dynamic environment will change the IMU's error coefficient such as installation error,scale factor and etc, which is calibrated in pure static environment.[1-3] Most aircraft navigation error model didn't consider the dynamic error, so the navigation calculation will hold that the sensitive value of the IMU is complete accurate and directly introduce measurement error to the model.

From elasticity point of view, the noumenon of the IMU has infinite degree of freedom, even if we just consider the motion freedom on the orthogonal axis, the coupling of rotational motion and angular motion will make the problem difficult.[4,5] Besides, the real inertial instruments must have sizes and shapes, they can't install exactly in the mass center of the noumenon of the IMU as a "point" in theory, it will lead to output error of the accelerometer if angular motion exist, this error is called "inner" lever arm effct. As same as the inner lever arm effct, the noumenon of the IMU can't install exactly in the mass center of the aircraft, which will lead to "external" lever arm effect when angular motion exist. If the lever arm vector is known, the error could be compensated by mechanics method or digital filtering method, and without the established lever arm, some dynamic online calibration methods are carried out through the remaining prior information, but all the reasearch didn't analyze the root cause of the error in dynamic environment.[6-10] On the basis of these, with some reasonable assuptions, the IMU is equivalent to Timoshenko beam and damper spring hybrid system, it is not lost in general that the cross section center of the beam does not coincide with the shear center, the differential equations of the hybrid system were decoupled and solved by the Timoshenko-beam's Green function, in order to establish the relationship between complex coupled vibration and lever arm effect.

\section{Equivalent model of the Hybrid System}

For the IMU installation, stent with rubber cushion system is generally used for fixing to aircraft. Although the rubber mat has infinite degree of freedom, but navigation system only need to focus 
on the response of inertial instrument sensitive axis direction. In the case of lateral-torsional coupling vibration, the stent of IMU is equivalent to Timoshenko beam, the rubber cushion system is equivalent to line spring(response lateral vibration), torsional spring(response torsional vibration) and damper, the noumenon of the IMU is equivalent to oscillator, assuming the system is stimulated by lateral force along the beam $p(x, t)$ and torsional force around the shear center $q(x, t)$, and the cross section center of the beam does not coincide with the shear center, the distance between the two is $\chi_{\alpha}$, so the hybird system is shown as Fig.1.

All manuscripts must be in English, also the table and figure texts, otherwise we cannot publish your paper. Please keep a second copy of your manuscript in your office. When receiving the paper, we assume that the corresponding authors grant us the copyright to use the paper for the book or journal in question. Should authors use tables or figures from other Publications, they must ask the corresponding publishers to grant them the right to publish this material in their paper.

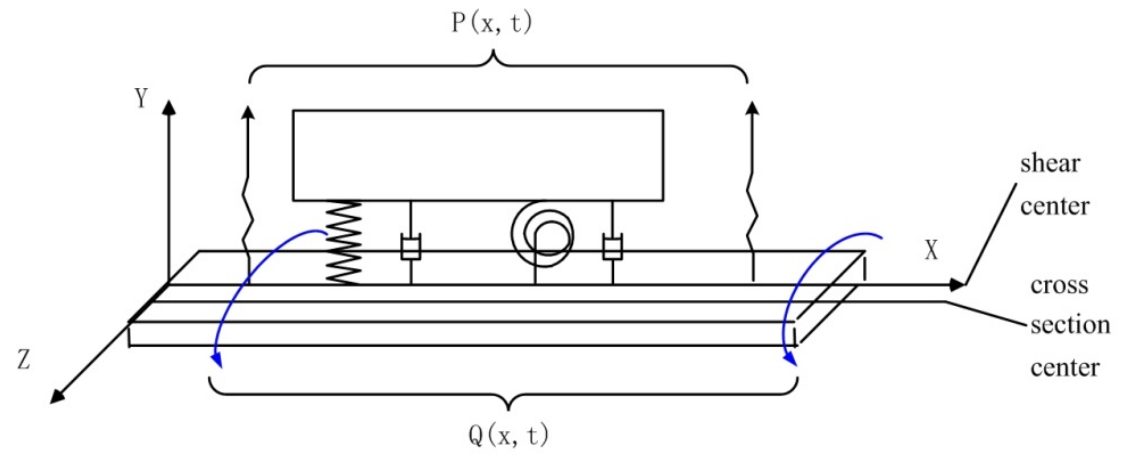

FIGURE 1. The equivalent hybrid system.

\section{Motion Differential Equations of the Hybrid System}

Because the Timoshenko beam itself is a continuum, when the cross section center and the shear center are not overlapping, transverse vibration and torsional vibration will be coupled, and there will be elastic force and damping force in the spring and beam joints[11,12]. Learn the classic theory of Timoshenko beam and according to the characteristics of the hybrid system, the beam differential equations and the spring differential equations could be combined with the nature of $\delta$ function. Learn from Reference[13] and reference[14], established the motion equations of the Timoshenko beam by the Hamilton principle, the motion differential equations of the hybrid system are

$$
\begin{aligned}
& E I \theta^{\prime \prime}+k G A\left(w^{\prime}-\theta\right)-\rho I \ddot{\theta}-c_{3} \dot{\theta}=0 \\
& k G A\left(w^{\prime \prime}-\theta^{\prime}\right)-m\left(\ddot{w}-\chi_{\alpha} \ddot{\psi}\right)-c_{1} \dot{w}=p(x, t)+F_{i}^{v}(t) \delta\left(x-x_{i}^{v}\right) \\
& m_{i} \ddot{w}_{i}+c_{i}^{v} \dot{w}_{i}+k_{i}^{v} w_{i}=c_{i}^{v} \dot{w}+k_{i}^{v} w \\
& G J \psi^{\prime \prime}-I_{\alpha} \ddot{\psi}+m_{\alpha} \ddot{w}-c_{2} \dot{\psi}=q(x, t)+F_{i}^{r}(t) \delta\left(x-x_{i}^{r}\right) \\
& J_{i} \ddot{\psi}_{i}+c_{i}^{r} \dot{\psi}_{i}+k_{i}^{r} \psi_{i}=c_{i}^{r} \dot{\psi}+k_{i}^{r} \psi
\end{aligned}
$$

where superscript (•) means taking the derivative of the time, superscript (') means taking the derivative of the position, $c_{1} 、 c_{2} 、 c_{3}$ is the flexural vibration damping, cross section rotating damping and torsional vibration damping of the beam, $E I$ is the bending stiffness of the beam, $k G A$ is the cross section rotational stiffness of the beam, $G J$ is the shear stiffness of the beam, $\theta$ is the beam section rotation angle which is caused by lateral vibration, $w$ is the displacement in lateral vibration, $\psi$ is the angle in torsional vibration, $\rho I$ is the moment of inertia of the beam, $m$ is the density of the beam, $I_{\alpha}$ is the moment of inertia of the beam per unit length, $m_{i}$ is the quality of the oscillator, $w_{i}$ is the displacement of the oscillator in lateral vibration, $c_{i}^{v}$ is the damping coefficient of the oscillator in lateral vibration, $k_{i}^{v}$ is the stiffness coefficient of the line spring, $x_{i}^{v}$ is the installation position of the line spring, $J_{i}$ is the moment of inertial of the oscillator, $\psi_{i}$ is the torsional angle of the oscillator, $c_{i}^{r}$ is the damping coefficient of the oscillator in torsional vibration, $k_{i}^{r}$ is the stiffness coefficient of the torsional spring, $x_{i}^{r}$ is the installation position of the torsional spring, $\delta()$ is the Dirac $\delta$ function, $F_{i}^{v} 、 F_{i}^{r}$ is the constraining force of lateral vibration and 
torsional vibration, respectively, and they are

$$
\begin{aligned}
& F_{i}^{v}(t)=c_{i}^{v}\left(\dot{w}_{i}-w\left(x_{i}^{v}, t\right)\right)+k_{i}^{v}\left(w_{i}-w\left(x_{i}^{v}, t\right)\right)=-m_{i} \ddot{w}_{i} \\
& F_{i}^{r}(t)=c_{i}^{r}\left(\dot{\psi}_{i}-\dot{\psi}\left(x_{i}^{r}, t\right)\right)+k_{i}^{r}\left(\psi_{i}-\psi\left(x_{i}^{r}, t\right)\right)=-J_{i} \ddot{\psi}_{i}
\end{aligned}
$$

\section{Simplification of the Differential Equations}

Although there are classical theory of the vibration for Timoshenko beam, but the equivalent hybrid system under the condition of lateral-torsional coupling vibration with multiple damping, such as damping of the oscillator in lateral vibration, flexural vibration damping of the beam, etc. When $\chi_{\alpha}$ is not zero, the vibration response in different directions could be coupled, so it is very difficult to solve the equations directly and must be simplified.

First, equation(2) is transformed, lateral vibration displacement $w$ is expressed in $\theta$

$$
\theta^{\prime}=w^{\prime \prime}-\frac{m}{k G A}\left(\ddot{w}-\chi_{\alpha} \ddot{\psi}\right)-\frac{c_{1}}{k G A} \dot{w}-\frac{1}{k G A}\left(p(x, t)-F_{i}^{v}(t) \delta\left(x-x_{i}^{v}\right)\right)
$$

Equation(1) takes the derivative with respect to displacement, it is

$$
E I \theta^{\prime \prime \prime}+k G A\left(w^{\prime \prime}-\theta^{\prime}\right)-\rho I \ddot{\theta}^{\prime}-c_{3} \dot{\theta}^{\prime}=0
$$

Using the method of separating variables, assuming that

$$
\left\{\begin{array}{l}
p(x, t)=P(x) e^{i \Omega t} \\
q(x, t)=Q(x) e^{i \Omega t} \\
w(x, t)=W(x) e^{i \Omega t} \\
\psi(x, t)=\Psi(x) e^{i \Omega t}
\end{array}\right.
$$

Substituting (8) and (10) into(9), the beam section rotation angle $\theta$ cancel out, (1) and (2) merged into

$$
W^{\prime \prime \prime \prime}+a_{1} W^{\prime \prime}+a_{2} W+a_{3} \Psi^{\prime \prime}+a_{4} \Psi=b_{1}\left(P(x)+F_{i}^{v} \delta\left(x-x_{i}^{v}\right)\right)^{\prime \prime}-b_{2}\left(P(x)+F_{i}^{v} \delta\left(x-x_{i}^{v}\right)\right)
$$

where the coefficients are

$$
\left\{\begin{array}{l}
a_{1}=-\left[i \Omega\left(\frac{c_{1}}{k G A}+\frac{c_{3}}{E I}\right)-\Omega^{2}\left(\frac{m}{k G A}+\frac{\rho I}{E I}\right)\right] \\
a_{2}=\frac{i \Omega c_{1}}{E I}-\Omega^{2}\left(\frac{m}{E I}+\frac{c_{1} C_{3}}{k G A \cdot E I}\right)- \\
i \Omega^{3}\left(\frac{m c_{3}}{k G A \cdot E I}+\frac{c_{1} \rho I}{k G A \cdot E I}\right)+\Omega^{4} \frac{m \rho I}{k G A \cdot E I} \\
a_{3}=-\frac{\Omega^{2} m \chi_{a}}{k G A} \\
a_{4}=\frac{m \chi_{a}}{E I}\left(\Omega^{2}+\frac{i \Omega^{3} c_{3}}{k G A}-\frac{\Omega^{4} \rho I}{k G A}\right) \\
b_{1}=\frac{1}{k G A} \\
b_{2}=\frac{1}{E I}+\frac{i \Omega c_{3}}{k G A \cdot E I}-\frac{\Omega^{2} \rho I}{k G A \cdot E I}
\end{array}\right.
$$

In the same way, Substituting (10) into(4) to separate variables, and (4) is simplified to

$$
\Psi^{\prime \prime}+a_{5} \Psi-a_{6} W=b_{3}\left(Q(x)+F_{i}^{r}(t) \delta\left(x-x_{i}^{r}\right)\right)
$$

where the coefficients are

$$
\left\{\begin{array}{l}
a_{5}=\frac{\Omega^{2} I_{\alpha}}{G J}-\frac{i \Omega c_{2}}{G J} \\
a_{6}=\frac{\Omega^{2} m \chi_{\alpha}}{G J} \\
b_{3}=-\frac{1}{G J}
\end{array}\right.
$$

\section{Modal Analysis of the Hybrid System}

For the hybrid system, it is very difficult to solve the vibration response determinately, but in 
terms of its physical background, the external excitation generated by aircraft during flight can be expressed in a random process, so we expect to solve the digital characteristics of vibration response according to the theory of random vibration.

The spring interacts with the beam only in the spring installation position, the differential equation means to solve the response which is caused by excitation force in a point on the beam, namely, the Green function of beam vibration. Under the lateral-torsional coupling excitation condition, the Green function should include lateral excitation force caused displacement function $G_{11}$, lateral excitation force caused torsional function $G_{12}$, torsional excitation force caused displacement function $G_{21}$ and torsional excitation force caused torsional function $G_{22}$.

\section{Intrinsic Mode Functions}

According to the derivation of reference[15][16], lateral excitation force caused displacement function $G_{11}$ is

$$
\begin{aligned}
& G_{11}\left(x, x_{i}^{v}\right)=H\left(x-x_{i}^{v}\right) \phi_{11}\left(x-x_{i}^{v}\right)+\phi_{12}(x) W(0)+\phi_{13}(x) W^{\prime}(0)+ \\
& \phi_{14}(x) W^{\prime \prime}(0)+\phi_{15}(x) W^{\prime \prime \prime}(0)+\phi_{16}(x) \Psi(0)+\phi_{17}(x) \Psi^{\prime}(0)
\end{aligned}
$$

Where $H(\cdot)$ is the Heaviside function, the expressions of coefficients $\phi_{i j}$ are detailed in reference [17], $W(0) 、 W^{\prime}(0) 、 W^{\prime \prime}(0) 、 W^{\prime \prime \prime}(0)$ and $\Psi(0) 、 \Psi^{\prime}(0)$ can be obtained by specific boundary conditions.

Green function $G_{11}$ means the response when all the lateral excitation force is concentrated in $x_{i}^{v}$, and is the solution of hybrid system lateral displacement $w$ for equation(14).

$$
W^{\prime \prime \prime \prime}+a_{1} W^{\prime \prime}+a_{2} W+a_{3} \Psi^{\prime \prime}+a_{4} \Psi=b_{1} \delta^{\prime \prime}\left(x-x_{i}^{v}\right)-b_{2} \delta\left(x-x_{i}^{v}\right)
$$

Intrinsic mode functions can be obtained under undamped free vibration conditions, therefore, all the damping and excitation in the previous article could be zero, and (3) transform as

$$
m_{i} \ddot{w}_{i}+k_{i}^{v} w_{i}=k_{i}^{v} w
$$

Using the method of separating variables, assuming that

$$
w_{i}(t)=B_{i} W\left(x_{i}\right) e^{i \Omega t}
$$

where $W\left(x_{i}\right)$ is the unknown intrinsic mode function.

Substituting (6), (10) and (16) into(11) and (15), in the case of all damping and excitation is zero, equation simplifies to

$$
\begin{aligned}
& W^{\prime \prime \prime \prime}+a_{1} W^{\prime \prime}+a_{2} W+a_{3} \Psi^{\prime \prime}+a_{4} \Psi=b_{1}\left(m_{i} B_{i} W\left(x_{i}\right) e^{i \Omega t} \Omega^{2} \cdot \delta\left(x-x_{i}^{v}\right)\right)^{\prime \prime}- \\
& b_{2}\left(m_{i} B_{i} W\left(x_{i}\right) e^{i \Omega t} \Omega^{2} \cdot \delta\left(x-x_{i}^{v}\right)\right)
\end{aligned}
$$

where $B_{i}=k_{i}^{v} / k_{i}^{v}-m_{i}$.

According to the physical meaning of the Green function, (17) is expressed as

$$
\begin{aligned}
& W^{\prime \prime \prime \prime}+a_{1} W^{\prime \prime}+a_{2} W+a_{3} \Psi^{\prime \prime}+a_{4} \Psi= \\
& b_{1}\left(m_{i} B_{i} W\left(x_{i}\right) e^{i \Omega t} \Omega^{2} \cdot G_{11}\right)^{\prime \prime}- \\
& b_{2}\left(m_{i} B_{i} W\left(x_{i}\right) e^{i \Omega t} \Omega^{2} \cdot G_{11}\right)
\end{aligned}
$$

When $G_{11}$ is known, according (18), we can obtain the $n$ order intrinsic mode function $W(x)$ of the hybrid system lateral vibration.

In the same way, torsional excitation force caused torsional function $G_{22}$ is

$$
\begin{aligned}
& G_{22}\left(x, x_{i}^{r}\right)=H\left(x-x_{i}^{r}\right) \phi_{41}\left(x-x_{i}^{r}\right)+\phi_{22}(x) W(0) \\
& +\phi_{23}(x) W^{\prime}(0)+\phi_{24}(x) W^{\prime \prime}(0)+\phi_{25}(x) W^{\prime \prime \prime}(0)+\phi_{26}(x) \Psi(0)+\phi_{27}(x) \Psi^{\prime}(0)
\end{aligned}
$$

$G_{22}$ means the response when all the torsional excitation force is concentrated in $x_{i}^{r}$, and is the solution of hybrid system torsional angle $\psi$ for equation(20).

$$
\Psi^{\prime \prime}+a_{5} \Psi-a_{6} W=b_{3} \delta\left(x-x_{i}^{r}\right)
$$

When $G_{22}$ is known, using the same method of separating variables, we we can obtain the $n$ order intrinsic mode function $\Psi(x)$ of the hybrid system torsional vibration.

\section{Complex Mode Analysis for Forced Vibration}

Under the condition of forced vibration, we generically consider the system damping is nonclassical, namely, non-proportional damping. Assuming that 


$$
\left\{\begin{array}{l}
w(x, t)=\sum_{n=1}^{N} \alpha_{n}(t) W_{n}(x)=W^{T}(x) \boldsymbol{\alpha}(t) \\
w_{i}(t)=\sum_{n=1}^{N} \alpha_{n}(t) B_{i n} W_{n}\left(x_{i}\right)=\boldsymbol{Y}^{T}\left(x_{i}\right) A_{i} \boldsymbol{\alpha}(t) \\
A_{i}=\operatorname{diag}\left[B_{i n}\right]
\end{array}\right.
$$

In(21), $N$ denotes intercept pre-n-order of the mode function to calculate approximately, $B_{i n}$ is the relationship between beam displacement and oscillator displacement, $\alpha_{n}(t)$ is the modal response of the system.

Because the damping of the system is not classical, complex mode analysis must be performed. We can obtain $2 \mathrm{~N}$ conjugate characteristic values and characteristic vectors according characteristic equations of the system, characteristic values matrix denotes as $\mathbf{p}$, the modal matrix composed of charactristic vectors denotes as $\mathbf{u}$, using expanded order method for complex modal transform of $\alpha_{n}(t)$, we can obtain the impulse response matrix of the system $\mathbf{h}(t)$, according to the Duhamel integral, the complex modal response is expressed as

$$
\mathbf{z}(t)=\int_{0}^{+\infty} \mathbf{h}(\omega) \mathbf{Q}(t-\omega) d \omega
$$

where $\mathbf{Q}(\cdot)$ is the generalized excitation after complex modal transform.

According to (22), we can obtain the covariance function of the complex modal response $C_{z}(t)$, and make inverse transformation of $\alpha_{n}(t)$ and $\mathbf{z}$, the covariance function of modal response $\alpha_{n}(t)$ is

$$
\boldsymbol{C}_{a}(\tau)=\boldsymbol{u} \boldsymbol{C}_{z}(t) \boldsymbol{u}^{* T}
$$

Assuming the external excitation is zero-mean and stationary, the covariance function of displacement response of the oscillator lateral vibration is

$$
C_{w_{i} w_{i}}(\tau)=\boldsymbol{Y}^{T}\left(x_{i}^{v}\right) \boldsymbol{A}_{i} \boldsymbol{C}_{\alpha}(\tau) \boldsymbol{A}_{i} \boldsymbol{Y}\left(x_{i}^{v}\right)
$$

The covariance function of speed response and acceleration response of the oscillator lateral vibration is

$$
\begin{aligned}
& C_{\dot{v}_{i} \dot{w}_{i}}(\tau)=\boldsymbol{Y}^{T}\left(x_{i}^{v}\right) \boldsymbol{A}_{i} \boldsymbol{p} \boldsymbol{C}_{\alpha}(\tau) \boldsymbol{p}^{*} \boldsymbol{A}_{i} \boldsymbol{Y}\left(x_{i}^{v}\right) \\
& C_{\ddot{v}_{i} \dot{w}_{i}}(\tau)=\boldsymbol{Y}^{T}\left(x_{i}^{v}\right) \boldsymbol{A}_{i} \boldsymbol{p} \boldsymbol{p} \boldsymbol{C}_{\alpha}(\tau) \boldsymbol{p}^{*} \boldsymbol{p}^{*} \boldsymbol{A}_{i} \boldsymbol{Y}\left(x_{i}^{v}\right)
\end{aligned}
$$

In the case of $\tau=0$, (24) and (26) are displacement variance and acceleration variance of the oscillator lateral vibration.

In the same method, we can obtain the torsional angle variance and torsional speed variance of the oscillator torsional vibration, they are

$$
\begin{aligned}
& C_{\psi_{i} \psi_{i}}(\tau)=\tilde{\boldsymbol{Y}}^{T}\left(x_{i}^{r}\right) \tilde{\boldsymbol{A}}_{i} \tilde{\boldsymbol{C}}_{\alpha}(\tau) \tilde{\boldsymbol{A}}_{i} \tilde{\boldsymbol{Y}}\left(x_{i}^{r}\right) \\
& C_{\dot{\psi}_{i} \dot{\psi}_{i}}(\tau)=\tilde{\boldsymbol{Y}}^{T}\left(x_{i}^{v}\right) \tilde{\boldsymbol{A}}_{i} \tilde{\boldsymbol{p}} \tilde{\boldsymbol{C}}_{\alpha}(\tau) \tilde{\boldsymbol{p}}^{*} \tilde{\boldsymbol{A}}_{i} \tilde{\boldsymbol{Y}}\left(x_{i}^{v}\right)
\end{aligned}
$$

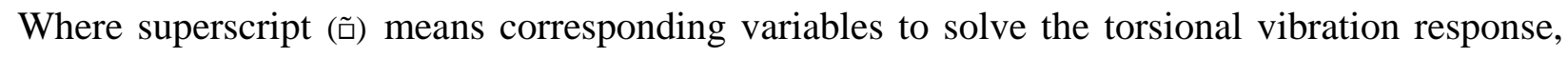
the specific method is no longer detailed.

\section{The Influence Mechanism of Vibration Response on Lever Arm Effect}

Through the previous analysis, it can be seen that when the noumenon of the IMU subject to external excitation, there will be minute lateral and torsional vibration around the static installation position. In order to make the problem analysis more pertinent, assuming that: the lever arm vector is known, the hybrid system got one lateral distribution force and one torsional force, there is absolute rigidity inside the noumennon, so inner inertial instruments will not affected by external excitation, all accelerometers installed in the center of mass of the noumennon ideally, it means on the premise of ignoring the inner lever arm effect to analysis the fundamental mechanism of the navigation error caused by lateral-torsional coupling vibration.

The Influence on Fixed Geometric Relationship of the Lever Arm Effect

When the aircraft appears in angular motion, the basic principle of the external lever arm effect 
is shown in Figure 2.[18]

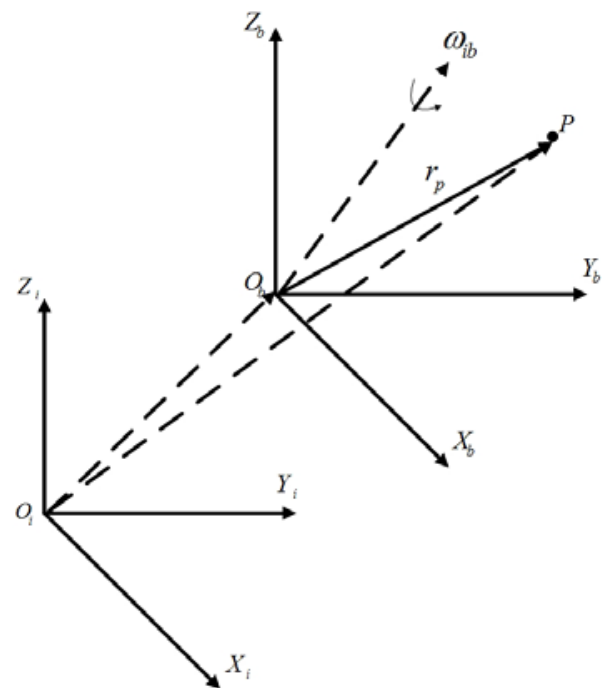

FIGURE 2 External lever arm effect sketch map

In Fig.2, $O_{i}$ is the origin of the inertial coordinate system, $O_{b}$ is the origin of the vehicle coordinate system(the navigation reference point), $P$ is the center of mass of the IMU noumenon, $\mathbf{r}_{p}$ is the external lever arm vector, $\omega_{i b}$ is the angular velocity of the aircraft.

According to kinematics principle, the relationship of specific force between accelerometer position and navigation reference point is

$$
a_{p}=a_{b}+\dot{\omega}_{i b} \times r_{p}+\omega_{i b} \times\left(\omega_{i b} \times r_{p}\right)
$$

where $\boldsymbol{\alpha}_{\boldsymbol{p}}$ denotes the specific force of the accelerometer position, $\boldsymbol{a}_{\boldsymbol{b}}$ denotes the specific force of the navigation reference point, the difference between the two is external lever arm effect $\Delta a$

$$
\Delta a=\dot{\omega}_{i b} \times r_{p}+\omega_{i b} \times\left(\omega_{i b} \times r_{p}\right)
$$

If the angular velocity is measured accurately, when the lever arm vector is known, the lever arm effect $\boldsymbol{\Delta a}$ can be used as a constant fully compensation in theory. But under the dynamic environment, the aircraft continues to be subject to external excitation, and the IMU noumenon will also continue to generate dynamic responses, so the IMU noumenon center of mass $P$ is always changing, and the external lever arm vector $\boldsymbol{r}_{\boldsymbol{p}}$ is no longer a deterministic vector.

An example is when the hybrid system get one lateral distribution force and one torsional force, the noumenon will shift with the lateral vibration while rotate around the axis, it is shown in Figure 3.

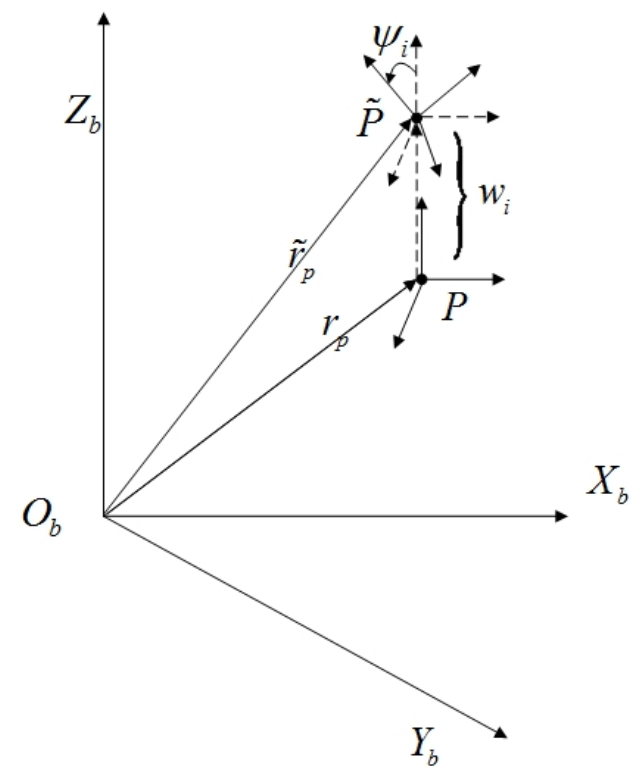

FIGURE 3 Dynamic response sketch map 
Where $\tilde{P}$ represents the IMU noumenon center of mass position of a transient state in response, $\tilde{r}_{p}$ denotes the real external lever arm vector at this moment, the center of mass in static environment shift $w_{i}$ along the lateral vibration to $\tilde{P}$ and rotate $\psi_{i}$ around the torsional axis, so the real lever arm effect should be

$$
\Delta a=\dot{\omega}_{i b} \times\left(r_{p}+\Delta r\right)+\omega_{i b} \times\left(\omega_{i b} \times\left(r_{p}+\Delta r\right)\right)
$$

Where $\boldsymbol{\Delta r}$ is the variation of the external lever arm vector.

Think in general terms, the external lever arm vector should change around static values, that is

$$
E(\Delta a)=\left|\dot{\omega}_{i b} \times r_{p}+\omega_{i b} \times\left(\omega_{i b} \times r_{p}\right)\right|
$$

According (24), with the covariance function of displacement response of the oscillator, we can obtain the variance of the external lever arm vector variation, it is

$$
\operatorname{Var}(\Delta \boldsymbol{r})=Y^{T}\left(x_{1}\right) A_{1} C_{\alpha}(0) A_{1} Y\left(x_{1}\right)
$$

Combining variance property, the variance of error caused by external lever arm changing is

$$
\operatorname{Var}(\boldsymbol{\Delta a})=\left|C_{1} \dot{\boldsymbol{\omega}}_{i b}{ }^{2} \operatorname{Var}(\boldsymbol{\Delta} \boldsymbol{r})+C_{2} \boldsymbol{\omega}_{i b}{ }^{4} \operatorname{Var}(\boldsymbol{\Delta r})\right|
$$

Where $C_{1}$ and $C_{2}$ denote the constant coefficients determined by $\dot{\omega}_{i b}, \omega_{i b}, \Delta r$ position relation.

Although the simultaneous rotation of the noumenon will not change the lever arm vector, but will cause a change in the sensitive direction of the accelerometers, which is shown in Figure 4.

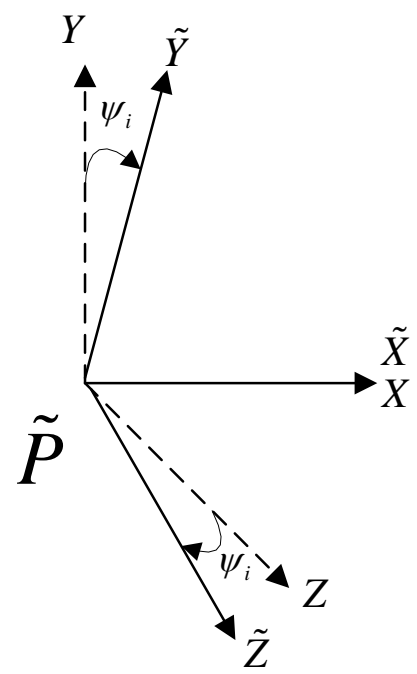

FIGURE 4 X-rotation of accelerometers sensitive direction

In Fig.4, $\tilde{P}-\tilde{X} \tilde{Y} \tilde{Z}$ is the real noumenon coordinate system, $\tilde{P}-X Y Z$ is the noumenon coordinate system in theory.

In the static environment, the specific force sensitved in the noumenon coordinate system convert to geographic coordinate system is denote as

$$
\mathbf{a}^{t}=C_{b}^{t} \mathbf{a}^{b}
$$

Where $C_{b}^{t}$ is the transformation matrix of the noumenon coordinate system to the geographic coordinate system, $\mathbf{a}^{b}$ is the specific force sensitved in the noumenon coordinate system, $\mathbf{a}^{t}$ is the specific force changed to geographic coordinate system.

But in the case of dynamic environment, the noumenon will rotate, so the real specific force sensitved in the noumenon coordinate system convert to geographic coordinate system is

$$
\tilde{\mathbf{a}}^{t}=C_{b}^{t} C_{\tilde{p}}^{p} \mathbf{a}^{b}
$$

Where $C_{\tilde{p}}^{p}$ is the transformation matrix of the real noumenon coordinate system to the noumenon coordinate system in theory, and

$$
C_{\tilde{p}}^{p}=\left(\begin{array}{ccc}
1 & 0 & 0 \\
0 & \cos \psi_{i} & \sin \psi_{i} \\
0 & -\sin \psi_{i} & \cos \psi_{i}
\end{array}\right)
$$

The resulting specific force conversion error is

$$
\Delta \tilde{\mathbf{a}}^{t}=C_{b}^{t} C_{\tilde{p}}^{p} \mathbf{a}^{b}-C_{b}^{t} \mathbf{a}^{b}=C_{b}^{t}\left(C_{\tilde{p}}^{p}-I\right) \mathbf{a}^{b}
$$




\section{The Influence on Angular Velocity measurement}

In the lever arm effect compensation process, the angular velocity must be known, but when the hybrid system under the dynamic environment, the noumenon will generate rotational response, and the response angular velocity will be measured by gyroscope, this is equivalent of directly introducing the measurement error, expressed as

$$
\tilde{\omega}_{i b}=\omega_{i b}+\Delta \omega
$$

Where $\tilde{\boldsymbol{\omega}}_{i b}$ is the real angular velocity under dynamic environment, $\omega_{i b}$ is the angular velocity of the aircraft, $\Delta \omega$ is the error between the two.

In the case of angular velocity error, the more accurate lever arm effect should be

$$
\Delta a=\left(\dot{\omega}_{i b}+\Delta \dot{\omega}\right) \times\left(r_{p}+\Delta r\right)+\left(\omega_{i b}+\Delta \omega\right) \times\left(\left(\omega_{i b}+\Delta \omega\right) \times\left(r_{p}+\Delta r\right)\right)
$$

Because the external excitation is completely random during the flight, even using the idealized equivalent model and assuming excitation is a smooth process, we can only get the digital features expression such as (24), (25), although it can be based on the digital features to optimize the material structure and installation mode to make the dynamic response smaller, but the error cannot be separated by a parsed formula.

\section{The Simulation Example}

Assuming the IMU installed in the cabin is equivalent to hybrid system of Timoshenko beam and spring oscillator, the noumenon is equivalent to a oscillator with absolute rigidity, the external excitation of the system reduce to white noise ideally, combining flight trajectory to calculate.

\section{The Influence on Navigation Calculating of the Lever Arm Effect Caused by Vibration}

Establishing the dimensionless model with real aircraft parameters according to the method introduced, calculating the natural frequency and vibration mode, the mode is truncated to five order and making complex modal analysis. According (24), (27), we can obtain the approximate variance of displacement in lateral vibration and rotation angle in torsional vibration, the variance is on $10^{-3}$ order of magnitude, generate Gauss random numbers as error according the variance, add the error to the compensation of lever arm effect and make navigation simulation, the sampling interval is $0.02 \mathrm{~s}$, the simulation time is $1000 \mathrm{~s}$, the position error and velocity error are shown in Figure.5.

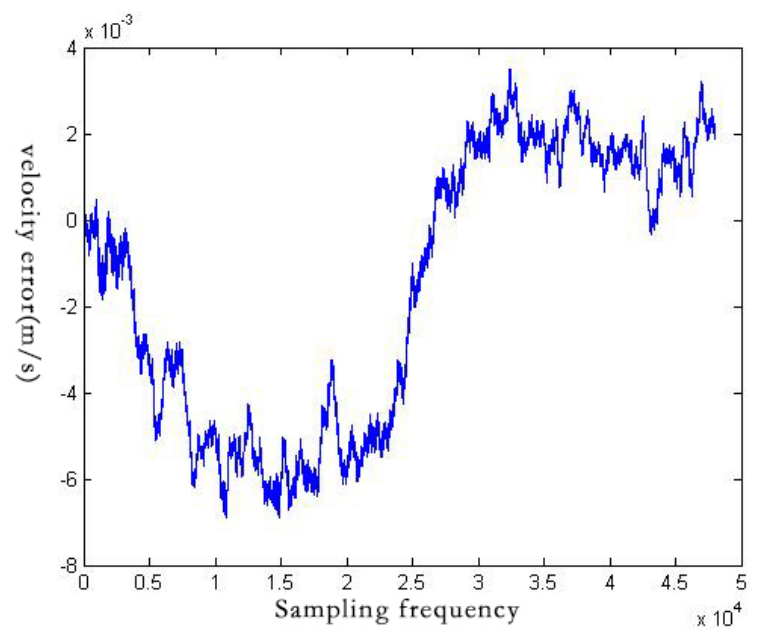

FIGURE 5 (a). Velocity error

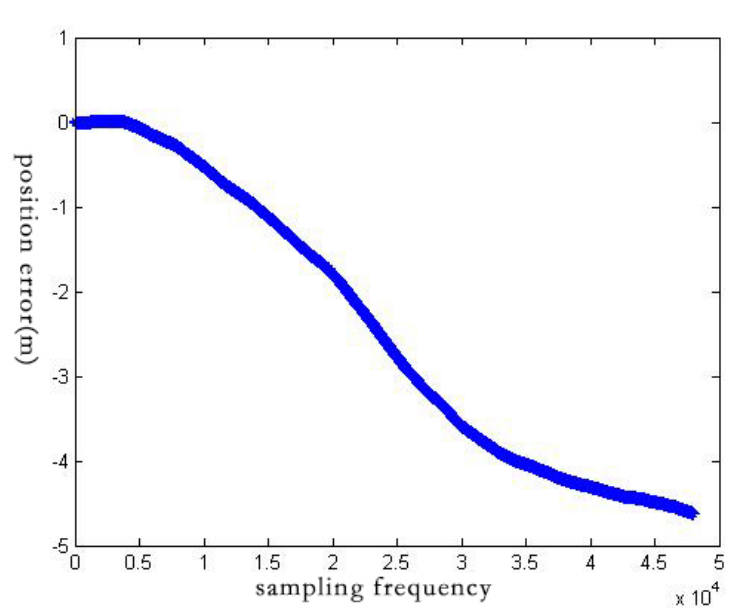

FIGURE 5 (b). Position error

The velocity and position have some errors in the navigation simulation, the maximum error of the velocity approximate $10^{-2}$ order of magnitude, and maximum error of position is close to $5 \mathrm{~m}$. Although the example is based on ideal conditions, there are still some difference between the dynamic excitation in actual flight, but suffice it to say that the incomplete compensation of the lever arm effect caused by vibration should be considered, especially when there is overload turn in flight or the lever arm is long. 
The Influence on Navigation Calculating of the Measurement Error Caused by Vibration

Getting one accelerometer and one laser gyro output as the data sample, part of the original pulse signal is shown in Figure.6.

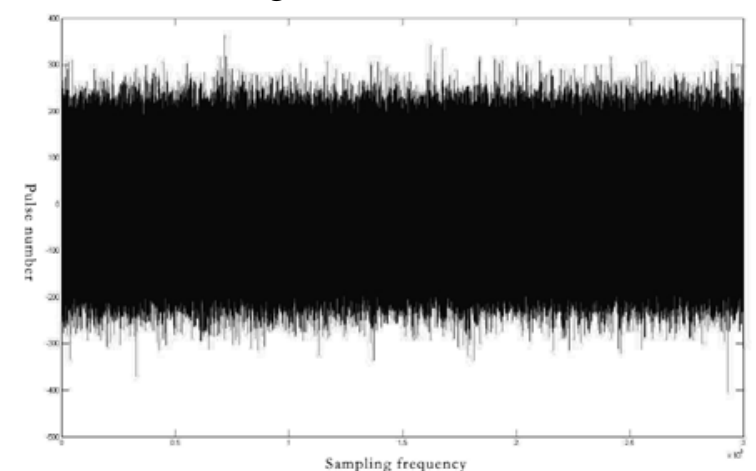

FIGURE 6 (a). Original pulse signal of gyro

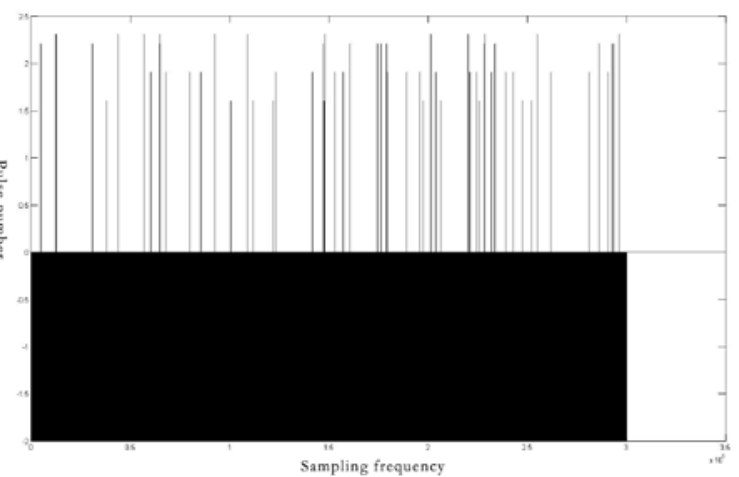

FIGURE 6 (b). Original pulse signal of accelerometer

According to the preceding method, getting the acceleration variance and the angular velocity variance of the noumenon vibration, Gaussian distribution random data is generated with the digital characteristics, the original pulse does the I/F conversion and superimpose with random data, then the superimposed data for navigation after the low-pass FIR filter, fight simulation continues 500 seconds, the position error on three axis is shown in Figrue.7.

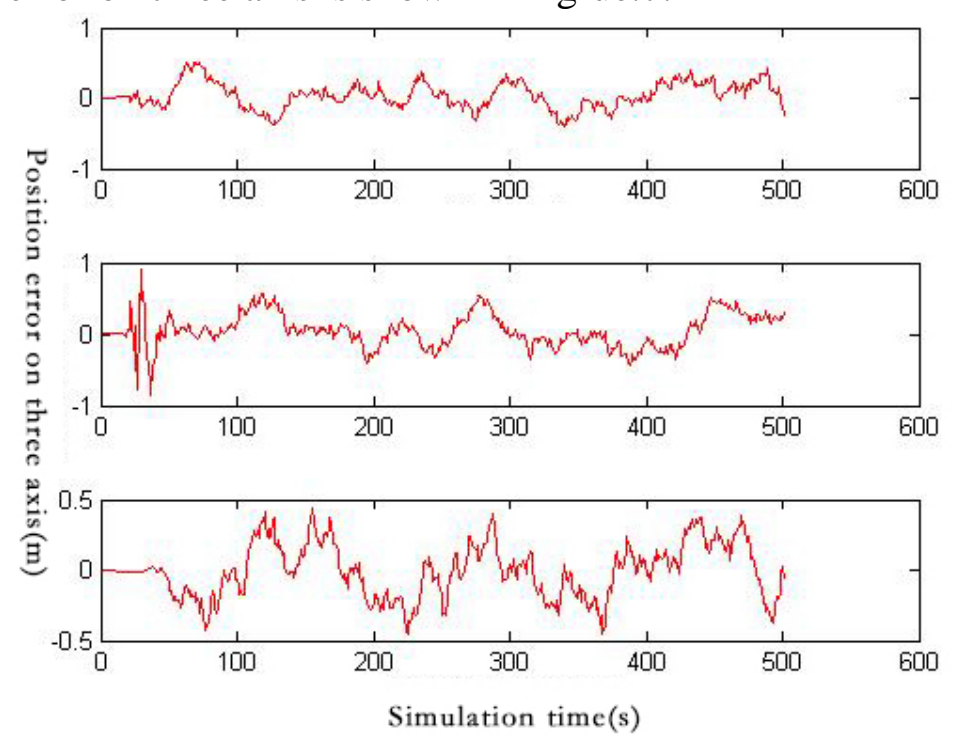

FIGURE 7. Position error on three axis

In the case of measurement error caused by dynamic response, we can see the max position error is less than one meter in Fig.7. The main reason on one hand is the navigation algorithm is based on acceleration and angular velocity increment, high frequency sampling will decrease the influence of this error, on the other hand the output of the inertial instrument must be filtered and transformed to digital signal, and set a certain band cut-off frequency and limit cut-off frequency, the error of high frequency vibration response is usually removed directly in the filter resistance band.

Therefore, when assuming absolute rigidity inside the noumennon and static calibration error coefficient is absolutely correct, the measurement error caused by the small vibration response is submerged in the background noise of the inertial instrument, the error may not be considered when aircraft with general precision requirement.

\section{Conclusion}

With the lateral-torsional coupling vibration, the IMU noumennon in the cabin could be equivalent to hybrid system consisting of Timoshenko beam ,damping spring and oscillator, the differential equation was solved by Green functions. 
The lever arm effect which is treated as deterministic vector in static condition will change with hybrid system response, and made the error incomplete compensated, but the measurement error caused by vibration was minimus quantity, it can be ignored when aircraft with general precision requirement.

If we can obtain the external excitation under actual working condition with multi-field coupling of heat, fluid, structure, etc, the analysis of vibration response influence will be more accurate.

All manuscripts must be in English, also the table and figure texts, otherwise we cannot publish your paper. Please keep a second copy of your manuscript in your office. When receiving the paper, we assume that the corresponding authors grant us the copyright to use the paper for the book or journal in question. Should authors use tables or figures from other Publications, they must ask the corresponding publishers to grant them the right to publish this material in their paper.

\section{References}

[1] Michael A. Blender, Heidi Wilkin. Flight dynamics of a hypersonic vehicle during inlet unstart[C]. 16th AIAA/DLR/DGLR International Space Planes and Hypersonic System and Technologies Conference, AIAA-2009-7292.

[2] Bo W, Zhi H D, Cheng L, et al. Estimation of information sharing error by Dynamic Deformation between inertial navigation system [J]. IEEE Transactions on Industrial Electronics, 2014,61(4):2015-2023.

[3] Raúl de Celis, Luis Cadarso, Jesús Sánchez. Guidance and control for high dynamic rotating artillery rockets[J]. Aerospace Science and Technology, 2017, 64(5): 204-212.

[4] Fu J B, Ma J, Yao J J. Coupling vibration study on the elastic supported inertial navigation system[J]. Structure and Environment engineering, 2005, 32(2): 46-51.

[5] Zhang Z X, Zhang D W. Theory analysis on resonance frequencies of linear vibration and torsional vibration of strapdown IMU damping system[J]. Journal of Chinese Inertial Technology, 2009,17(6):654-657.

[6] Yan G M, Yan W S, Xu D M. Analysis and compensation on inner lever arm effect of strapdown inertial measurement unit[J]. Journal of Chinese Inertial Technology, 2008, 16(2):148153.

[7] Zeng J L, Lei W, Kui L, et al. A compensation method of lever arm effect for tri-axis hybird inertial navigation system based on fiber optic gyro[J]. Measurement Science and Technology, 2017, 28(5):055103.

[8] Yuan L, Xiang H C. Random misalignment and lever arm vector online estimation in shipborne aircraft transfer alignment[J]. Measurement, 2014,47:756-764.

[9] Quan C, Mai Y Z, Yan Z. Dynamic lever arm compensation of SINS/GPS integrated system for aerial mapping [J]. Measurement, 2015, 60:39-49.

[10] Qian Z, Lei W, Zeng J L, et al. An accurate calibration method based on velocity in a rotational inertial navigation system[J]. Sensors, 2015, 15(8):18443-18458.

[11] Sapountzakis E J, Dourakopoulos J A. Shear deformation effect in flexural-torsional vibrations of beams by BEM[J]. Acta Mechanica, 2009, 203(3-4):197-221.

[12] De Borbon F, Mirasso A, Ambrosini D. A beam element for coupled torsional-flexural vibration of doubly unsymmetrical thin walled beams axially loaded[J]. Computers \& Structures, 2011, 89(13):1406-1416.

[13] Banerjee J R. Explicit modal analysis of an axially loaded Timoshenko beam with bendingtorsion coupling[J]. Journal of Applied Mechanics, 2000, 67(2):307-313.

[14] Banerjee J R, Williams F W. Coupled bending-torsional dynamic stiffness matrix of an axially loaded Timoshenko beam element[J]. International Journal of Solids and Structures, 1994,31(6):749-762.

[15] Foda M A, Abduljabbar Z. A dynamic Green function formulation for the response of a beam structure to a moving mass[J]. Journal of Sound and Vibration, 1998, 210(3):295-306.

[16] Li X Y, Zhao X, Li Y H. Green's functions of the forced vibration of Timoshenko beams with damping effect[J]. Journal of Sound and Vibration, 2014, 333(6):1781-1795. 
[17] Han H S. Study on the dynamical responses of beam structures using Green's functions[D]. Harbin Institute of Technology, 2016.

[18] Zhi X, Hui P, Jie W, et al. Dynamic Calibration Method for SINS Lever-Arm Effect for HCVs[J]. IEEE Transactions on Aerospace and electronic systems, 2015, 51(4): 2760-2770. 